\title{
INEQUALITIES FOR EIGENVALUES OF FOURTH ORDER ELLIPTIC OPERATORS IN DIVERGENCE FORM ON RIEMANNIAN MANIFOLDS
}

\author{
SHAHROUD AZAMI
}

\begin{abstract}
In this paper, we study eigenvalue of linear fourth order elliptic operators in divergence form with Dirichlet boundary condition on a bounded domain in a compact Riemannian manifolds with boundary (possibly empty) and find a general inequality for them. As an application, by using this inequality, we study eigenvalues of this operator on compact domains of complete submanifolds in a Euclidean space.
\end{abstract}

\section{INTRODUCTION}

In this paper, let $(M,\langle\rangle$,$) be an n$-dimensional complete Riemannian manifold and let $\Omega \subset M$ be a bounded connected domain with smooth boundary $\partial \Omega$ in $M$. Denote by $\Delta$ the Beltrami-Laplace operator on $M$. The study of the spectrum of geometric operator is an important topic and many works have been done in this area. The clamped plate problem or the Dirichlet biharmonic operator for a connected bounded domain $\Omega \subset \mathbb{R}^{n}$ is given by

$$
\begin{cases}\Delta^{2} u=\lambda u, & \text { in } \Omega \\ u=\frac{\partial u}{\partial \nu}=0 & \text { on } \partial \Omega,\end{cases}
$$

where $\nu$ is the outward unit normal vector field of $\partial \Omega$. Suppose that $\left\{\lambda_{i}\right\}_{i=1}^{\infty}$ is eigenvalues of the problem (1.1). Payn et'al proved in paper [4 that

$$
\lambda_{k+1}-\lambda_{k} \leq \frac{8(n+2)}{n^{2}} \frac{1}{k} \sum_{i=1}^{k} \lambda_{i}, \quad k=1,2, \ldots .
$$

In 1984, Hile and Yeh 2] generalized and showed

$$
\sum_{i=1}^{k} \frac{\lambda_{i}^{\frac{1}{2}}}{\lambda_{k+1}-\lambda_{i}} \geq \frac{n^{2} k^{\frac{3}{2}}}{8(n+2)}\left(\sum_{i=1}^{k} \lambda_{i}\right)^{-\frac{1}{2}}, \quad k=1,2, \ldots
$$

In 1990, Hook [3] obtained the following inequality

$$
\frac{n^{2} k^{2}}{8(n+2)} \leq\left(\sum_{i=1}^{k} \lambda_{i}^{\frac{1}{2}}\right)\left(\sum_{i=1}^{k} \frac{\lambda_{i}^{\frac{1}{2}}}{\lambda_{k+1}-\lambda_{i}}\right), \quad k=1,2, \ldots
$$

In 2006, Cheng and Yang [1] obtained the inequality

$$
\lambda_{k+1}-\frac{1}{k} \sum_{i=1}^{k} \lambda_{i} \leq\left(\frac{8(n+2)}{n^{2}}\right)^{\frac{1}{2}} \frac{1}{k} \sum_{i=1}^{k}\left(\lambda_{i}\left(\lambda_{k+1}-\lambda_{i}\right)\right)^{\frac{1}{2}}, \quad k=1,2, \ldots
$$

2010 Mathematics Subject Classification. 35P15; 35J93, 53C42.

Key words and phrases. Eigenvalue, Elliptic opeartor, Immersion. 
In 2007, Wang and Xia [5], proved universal bounds for eigenvalues of the biharmonic operator on Riemannian manifolds, for instance they showed that, when $\Omega$ is a compact domain in $\mathbb{R}^{n}$, then

$$
\lambda_{k+1} \leq \frac{1}{k} \sum_{i=1}^{k} \lambda_{i}+\left\{\frac{64}{n^{2} k^{2}}\left(\sum_{i=1}^{k} \lambda_{i}^{\frac{1}{2}}\right)\left(\sum_{i=1}^{k} \lambda_{i}^{\frac{3}{2}}\right)-\frac{1}{k} \sum_{i=1}^{k}\left(\lambda_{i}-\frac{1}{k} \sum_{i=1}^{k} \lambda_{i}\right)^{2}\right\}^{\frac{1}{2}}, k=1,2, \ldots
$$

The aim of the present work is to study the eigenvalues of linear fourth order elliptic operator in divergence form on Riemannian manifolds. In special case, this operator is the biharmonic operator. We prove some general inequalities for them. By using these inequalities, we obtain, when $\Omega$ is a compact domains of complete submanifolds in a Euclidean space.

Let $T$ be symmetric positive definite $(1,1)$-tensor on $M$ and $\Omega \subset M$ be a compact domain with smooth boundary $\partial \Omega$ in $M$. We will studying the eigenvalue problem

$$
\begin{cases}\mathcal{L}^{2} u=\lambda u, & \text { in } \Omega \\ u=\frac{\partial u}{\partial \nu}=0 & \text { on } \partial \Omega\end{cases}
$$

where $\mathcal{L} u=\operatorname{div}(T(\nabla u))$ and $\nabla$ is the gradient operator of $M$. If $T$ be the identity tensor the $\mathcal{L}=\Delta$. The main results of this paper are as follow

Theorem 1.1. Let $\Omega$ be a domain in an n-dimensional complete Riemannian manifold $(M,\langle\rangle$,$) isometrically immersed in \mathbb{R}^{m}, \lambda_{i}$ be the ith eigenvalue of (1.2) and $u_{i}$ be the corresponding orthonormal real-valued eigenfunction, that is

$$
\begin{cases}\mathcal{L}^{2} u_{i}=\lambda_{i} u_{i}, & \text { in } \Omega \\ u_{i}=\frac{\partial u_{i}}{\partial \nu}=0, & \text { on } \partial \Omega \\ \int_{\Omega} u_{i} u_{j} d m=\delta_{i j} & \forall i, j=1,2, \ldots\end{cases}
$$

Then for any positive constant $\delta$ and any positive integer $k$, we have

$$
\sum_{i=1}^{k}\left(\lambda_{k+1}-\lambda_{i}\right)^{2} \int_{\Omega} u_{i}^{2} \operatorname{tr}(T) d m \leq \delta \sum_{i=1}^{k}\left(\lambda_{k+1}-\lambda_{i}\right)^{2} A_{i}+\frac{1}{\delta} \sum_{i=1}^{k}\left(\lambda_{k+1}-\lambda_{i}\right) B_{i}
$$

where

$$
\begin{aligned}
A_{i}= & 2 \int_{\Omega} u_{i} \mathcal{L} u_{i}\langle\operatorname{tr}(\alpha \circ T)+\operatorname{tr}(\nabla T), I\rangle d m+2 \int_{\Omega} u_{i}\left\langle T\left(\nabla \mathcal{L} u_{i}\right), I\right\rangle d m \\
& +\int_{\Omega} u_{i}^{2}\left(\left.|| \operatorname{tr}(\alpha \circ T)\right|^{2}+|\operatorname{tr}(\nabla T)|^{2}\right) d m+4 \int_{\Omega} u_{i}\left\langle T\left(\nabla u_{i}\right), \operatorname{tr}(\nabla T)\right\rangle d m \\
& +4 \int_{\Omega}\left|T\left(\nabla u_{i}\right)\right|^{2} d m+2 \int_{\Omega} \mathcal{L} u_{i}\left\langle T\left(\nabla u_{i}\right), I\right\rangle d m
\end{aligned}
$$

and

$$
B_{i}=\int_{\Omega}\left\{\left|T\left(\nabla u_{i}\right)\right|^{2}+u_{i}\left\langle T\left(\nabla u_{i}\right), \operatorname{tr}(\nabla T)\right\rangle+\frac{u_{i}^{2}}{4}\left(|| \operatorname{tr}(\alpha \circ T) \|^{2}+|\operatorname{tr}(\nabla T)|^{2}\right)\right\} d m,
$$

where $I(x)=\left(x_{1}, \ldots, x_{m}\right)$ for any $x=\left(x_{1}, \ldots, x_{m}\right) \in \mathbb{R}^{n},\|f\|^{2}=\int_{\Omega} f^{2} d m$, dm is the volume form on $\Omega, \alpha$ is the fundamental form of $M$ and $\alpha \circ T=\alpha(T(),$.$) .$ 
Theorem 1.2. Let $\Omega$ be a domain in an $n$-dimensional complete Riemannian manifold $(M,\langle\rangle$,$) isometrically immersed in \mathbb{R}^{m}, \lambda_{i}$ be the ith eigenvalue of (1.2) and $u_{i}$ be the corresponding orthonormal real-valued eigenfunction. Then for any positive constant $\delta$ and any positive integer $k$, we have

$$
\sum_{i=1}^{k}\left(\lambda_{k+1}-\lambda_{i}\right)^{2} \int_{\Omega} u_{i}^{2} \operatorname{tr}(T) d m \leq \delta \sum_{i=1}^{k}\left(\lambda_{k+1}-\lambda_{i}\right)^{2} C_{i}+\frac{1}{\delta} \sum_{i=1}^{k}\left(\lambda_{k+1}-\lambda_{i}\right) D_{i}
$$

where

$$
\begin{aligned}
C_{i}= & 2\left(\sqrt{m-n} S_{0} T_{*}+T_{0}\right) I_{0} \lambda_{i}^{\frac{1}{2}}+I_{0}\left\|T\left(\nabla \mathcal{L} u_{i}\right)\right\|_{L^{2}(\Omega)}+(m-n) S_{0}^{2} T_{*}^{2}+T_{0}^{2} \\
& +4 T_{0}\left\|T\left(\nabla u_{i}\right)\right\|_{L^{2}(\Omega)}+4\left\|T\left(\nabla u_{i}\right)\right\|_{L^{2}(\Omega)}+2 \lambda_{i} I_{0}\left\|T\left(\nabla u_{i}\right)\right\|_{L^{2}(\Omega)}
\end{aligned}
$$

and

$$
D_{i}=\left\|T\left(\nabla u_{i}\right)\right\|_{L^{2}(\Omega)}+T_{0}\left\|T\left(\nabla u_{i}\right)\right\|_{L^{2}(\Omega)}+\frac{1}{4}\left((m-n) S_{0}^{2} T_{*}^{2}+T_{0}^{2}\right)
$$

where $S_{0}=\max \left\{\sup _{\bar{\Omega}}\left|S_{e_{k}}\right|: k=n+1, \ldots, m\right\}, S_{e_{k}}$ is the Weingarten operator of the immersion with respect to $e_{k}, T_{*}=\sup _{\bar{\Omega}}|T|, T_{0}=\sup _{\bar{\Omega}}|\operatorname{tr}(\nabla T)|$ and $I_{0}=$ $\sup _{\bar{\Omega}}|I|$.

Theorem 1.3. Let $\Omega$ be a domain in an $n$-dimensional complete Riemannian manifold $(M,\langle\rangle$,$) isometrically immersed in \mathbb{R}^{m}$ with mean curvature $H$ and $\lambda_{i}$ be the ith eigenvalue of biharmonic operator, that is

$$
\begin{cases}\Delta^{2} u_{i}=\lambda_{i} u_{i}, & \text { in } \Omega \\ u_{i}=\frac{\partial u_{i}}{\partial \nu}=0, & \text { on } \partial \Omega \\ \int_{\Omega} u_{i} u_{j} d m=\delta_{i j} & \forall i, j=1,2, \ldots\end{cases}
$$

Then for any positive constant $\delta$ and any positive integer $k$, we have

$$
\begin{aligned}
n \sum_{i=1}^{k}\left(\lambda_{k+1}-\lambda_{i}\right)^{2} \leq & \delta \sum_{i=1}^{k}\left(\lambda_{k+1}-\lambda_{i}\right)^{2}\left(2 n H_{0} I_{0} \lambda_{i}^{\frac{1}{2}}+n^{2} H_{0}^{2}+4 \lambda_{i}^{\frac{1}{2}}\right) \\
& +\frac{1}{\delta} \sum_{i=1}^{k}\left(\lambda_{k+1}-\lambda_{i}\right)\left(\lambda_{i}^{\frac{1}{2}}+\frac{1}{4} n^{2} H_{0}^{2}\right)
\end{aligned}
$$

where $H_{0}=\sup _{\bar{\Omega}}|H|$ and $I_{0}=\sup _{\bar{\Omega}}|I|$.

Corollary 1.4. Let $\Omega$ be a domain in an $n$-dimensional complete minimal Riemannian submanifold $(M,\langle\rangle$,$) in \mathbb{R}^{m}$ and $\lambda_{i}$ be the $i$ th eigenvalue of biharmonic operator. Then for any positive integer $k$, we have

$$
\begin{aligned}
\lambda_{k+1} \leq & \frac{1}{2 k}\left(2+\frac{1}{n^{2}}\right) \sum_{i=1}^{k} \lambda_{i} \\
& +\left\{\frac{1}{k^{2}}\left(1+\frac{8}{n^{2}}\right)^{2}\left(\sum_{i=1}^{k} \lambda_{i}\right)^{2}-\frac{1}{k}\left(1+\frac{16}{n^{2}}\right) \sum_{i=1}^{k} \lambda_{i}^{2}\right\}^{\frac{1}{2}},
\end{aligned}
$$

and

$$
\lambda_{2} \leq\left(1+\frac{17}{2 n^{2}}\right) \lambda_{1}
$$




\section{Preliminaries}

In this section, we describe the necessary tools about tensor $T$ and problem (1.2) which enable us to prove our results. Throughout the paper, for any vector fields $X, Y$, we denote $\langle T(X), Y\rangle$ with $T(X, Y)$. For any $u, v \in C^{\infty}(\Omega)$, straightforward computation implies that

$$
\mathcal{L}(u v)=v \mathcal{L} u+u \mathcal{L} v+2 T(\nabla u, \nabla v) .
$$

Let $d \mu$ be the volume form on the boundary induced by the outward normal vector field $\nu$ on $\partial \Omega$. The divergence theorem for operator $\mathcal{L}$ as follows

$$
\int_{\Omega} \mathcal{L} u d m=\int_{\partial \Omega} T(\nabla u, \nu) d \mu
$$

then the integration by parts yields

$$
\int_{\Omega} v \mathcal{L} u d m=-\int_{\Omega} T(\nabla u, \nabla v) d m+\int_{\partial \Omega} v T(\nabla u, \nu) d \mu .
$$

Hence, the operators $\mathcal{L}$ and $\mathcal{L}^{2}$ are self-adjoint operator in the space of all function in $L^{2}(\Omega, d m)$ that vanish on $\partial \Omega$. Therefore the eigenvalues of problem (1.2) are real and discrete.

Proposition 2.1. Let $\Omega$ be a domain in a an $n$-dimensional complete Riemannian manifold $(M,\langle\rangle),, \lambda_{i}$ be the $i$ th eigenvalue of (1.2) and $u_{i}$ be the corresponding orthonormal real-valued eigenfunction. Then for any $h \in C^{4}(\Omega) \cup C^{3}(\partial \Omega)$ and any positive integer $k$, we have

$$
\begin{aligned}
\sum_{i=1}^{k}\left(\lambda_{k+1}-\lambda_{i}\right)^{2} w_{i} \leq & \sum_{i=1}^{k}\left(\lambda_{k+1}-\lambda_{i}\right)\left\|p_{i}\right\|^{2} \\
\sum_{i=1}^{k}\left(\lambda_{k+1}-\lambda_{i}\right)^{2} v_{i} \leq & \delta \sum_{i=1}^{k}\left(\lambda_{k+1}-\lambda_{i}\right)^{2} w_{i} \\
& +\frac{1}{\delta} \sum_{i=1}^{k}\left(\lambda_{k+1}-\lambda_{i}\right)\left\|T\left(\nabla h, \nabla u_{i}\right)+\frac{u_{i} \mathcal{L} h}{2}\right\|^{2}, \\
\sum_{i=1}^{k}\left(\lambda_{k+1}-\lambda_{i}\right)^{2} v_{i} \leq & \delta \sum_{i=1}^{k}\left(\lambda_{k+1}-\lambda_{i}\right)\left\|p_{i}\right\|^{2} \\
& +\frac{1}{\delta} \sum_{i=1}^{k}\left(\lambda_{k+1}-\lambda_{i}\right)\left\|T\left(\nabla h, \nabla u_{i}\right)+\frac{u_{i} \mathcal{L} h}{2}\right\|^{2},
\end{aligned}
$$

where $\delta$ is any positive constant,

$$
\begin{aligned}
w_{i} & =\int_{\Omega} h u_{i} p_{i} d m, \\
p_{i} & =\mathcal{L} h \mathcal{L} u_{i}+2 T\left(\nabla h, \nabla \mathcal{L} u_{i}\right)+\mathcal{L}\left(u_{i} \mathcal{L} h\right)+2 \mathcal{L} T\left(\nabla h, \nabla u_{i}\right), \\
v_{i} & =\int_{\Omega} u_{i}^{2} T(\nabla h, \nabla h) d m .
\end{aligned}
$$

Proof. For each $i, 1 \leq i \leq k$, consider the functions $\phi_{i}: \Omega \rightarrow \mathbb{R}$ given by

$$
\phi_{i}=h u_{i}-\sum_{j=1}^{k} a_{i j} u_{j}
$$


where $a_{i j}=\int_{\Omega} h u_{i} u_{j} d m$. We have $\left.\phi_{i}\right|_{\partial \Omega}=\left.\frac{\partial \phi_{i}}{\partial \nu}\right|_{\partial \Omega}=0$ and

$$
\int_{\Omega} \phi_{i} u_{r} d m=\int_{\Omega} h u_{i} u_{r} d m-\sum_{j=1}^{k} a_{i j} \int_{\Omega} u_{r} u_{j} d \mu=0, \quad \forall i, r=1,2, \ldots, k .
$$

Then by the inequality of Rayleigh-Ritz, we get

$$
\lambda_{k+1} \leq \frac{\int_{\Omega} \phi_{i} \mathcal{L}^{2} \phi_{i} d m}{\int_{\Omega} \phi_{i}^{2} d m}, \quad \forall i=1,2, \ldots, k .
$$

Since

$$
\mathcal{L} \phi_{i}=\mathcal{L}\left(h u_{i}\right)-\sum_{j=1}^{k} a_{i j} \mathcal{L} u_{j}=h \mathcal{L} u_{i}+u_{i} \mathcal{L} h+2 T\left(\nabla h, \nabla u_{i}\right)-\sum_{j=1}^{k} a_{i j} \mathcal{L} u_{j},
$$

we obtain

$$
\mathcal{L}^{2} \phi_{i}=\mathcal{L} h \mathcal{L} u_{i}+\lambda_{i} h u_{i}+2 T\left(\nabla h, \nabla \mathcal{L} u_{i}\right)+\mathcal{L}\left(u_{i} \mathcal{L} h\right)+2 \mathcal{L} T\left(\nabla h, \nabla u_{i}\right)-\sum_{j=1}^{k} a_{i j} \lambda_{j} u_{j},
$$

therefore we get

$$
\int_{\Omega} \phi_{i} \mathcal{L}^{2} \phi_{i} d m=\lambda_{i}\left\|\phi_{i}\right\|^{2}+\int_{\Omega} h u_{i} p_{i} d m-\sum_{j=1}^{k} a_{i j} r_{i j}
$$

where $r_{i j}=\int_{\Omega} p_{i} u_{j} d m$ and

$$
p_{i}=\mathcal{L} h \mathcal{L} u_{i}+2 T\left(\nabla h, \nabla \mathcal{L} u_{i}\right)+\mathcal{L}\left(u_{i} \mathcal{L} h\right)+2 \mathcal{L} T\left(\nabla h, \nabla u_{i}\right) .
$$

Using integration by parts, we deduce that

$$
\begin{aligned}
& \int_{\Omega} u_{j} \mathcal{L} T\left(\nabla h, \nabla u_{i}\right) d m+\int_{\Omega} u_{j} T\left(\nabla h, \nabla \mathcal{L} u_{i}\right) d m \\
& =-\int_{\Omega} T\left(\nabla u_{j}, \nabla T\left(\nabla h, \nabla u_{i}\right)\right) d m-\int_{\Omega} \operatorname{div}\left(u_{j} T \nabla h\right) \mathcal{L} u_{i} d m \\
& =\int_{\Omega} \mathcal{L} u_{j} T\left(\nabla h, \nabla u_{i}\right) d m-\int_{\Omega} \mathcal{L} u_{i} T\left(\nabla h, \nabla u_{j}\right) d m-\int_{\Omega} u_{j} \mathcal{L} h \mathcal{L} u_{i} d m .
\end{aligned}
$$

On the other hand

$$
\begin{aligned}
\text { (2.8) } \int_{\Omega} \mathcal{L} u_{j} T\left(\nabla h, \nabla u_{i}\right) d m-\int_{\Omega} \mathcal{L} u_{i} T\left(\nabla h, \nabla u_{j}\right) d m \\
=-\int_{\Omega} h \operatorname{div}\left(\mathcal{L} u_{j} T \nabla u_{i}\right)+\int_{\Omega} h \operatorname{div}\left(\mathcal{L} u_{i} T \nabla u_{j}\right) \\
=-\int_{\Omega}\left\langle h \nabla \mathcal{L} u_{j}, T \nabla u_{i}\right\rangle d m+\int_{\Omega}\left\langle h \nabla \mathcal{L} u_{i}, T \nabla u_{j}\right\rangle d m \\
=\int_{\Omega} u_{i} \operatorname{div}\left(h T \nabla u_{j}\right) d m-\int_{\Omega} u_{j} \operatorname{div}\left(h T \nabla u_{i}\right) d m \\
=\int_{\Omega}\left(u_{i} h \mathcal{L}^{2} u_{j}-u_{j} h \mathcal{L}^{2} u_{i}\right) d m+\int_{\Omega}\left(\left\langle u_{i} T \nabla h, \nabla \mathcal{L} u_{j}\right\rangle-\left\langle u_{j} T \nabla h, \nabla \mathcal{L} u_{i}\right\rangle\right) d m \\
=\left(\lambda_{j}-\lambda_{i}\right) a_{i j}-\int_{\Omega} \mathcal{L} u_{j} T\left(\nabla h, \nabla u_{i}\right) d m+\int_{\Omega} \mathcal{L} u_{i} T\left(\nabla h, \nabla u_{j}\right) d m \\
\quad-\int_{\Omega} u_{i} \mathcal{L} u_{j} \mathcal{L} h d m+\int_{\Omega} u_{j} \mathcal{L} u_{i} \mathcal{L} h d m,
\end{aligned}
$$


which implies that

$$
\begin{aligned}
& 2 \int_{\Omega} \mathcal{L} u_{j} T\left(\nabla h, \nabla u_{i}\right) d m-2 \int_{\Omega} \mathcal{L} u_{i} T\left(\nabla h, \nabla u_{j}\right) d m \\
& =\left(\lambda_{j}-\lambda_{i}\right) a_{i j}-\int_{\Omega} u_{i} \mathcal{L} u_{j} \mathcal{L} h d m+\int_{\Omega} u_{j} \mathcal{L} u_{i} \mathcal{L} h d m .
\end{aligned}
$$

Substituting (2.7) into (2.9), we have

$$
\begin{aligned}
& 2 \int_{\Omega} u_{j} \mathcal{L} T\left(\nabla h, \nabla u_{i}\right) d m+2 \int_{\Omega} u_{j} T\left(\nabla h, \nabla \mathcal{L} u_{i}\right) d m \\
& =\left(\lambda_{j}-\lambda_{i}\right) a_{i j}-\int_{\Omega} u_{i} \mathcal{L} u_{j} \mathcal{L} h d m-\int_{\Omega} u_{j} \mathcal{L} u_{i} \mathcal{L} h d m .
\end{aligned}
$$

Moreover

$$
\int_{\Omega} u_{j} \mathcal{L}\left(u_{i} \mathcal{L} h\right) d m=\int_{\Omega} u_{i} \mathcal{L} u_{j} \mathcal{L} h d m .
$$

Combining (2.10), 2.11) and $r_{i j}=\int_{\Omega} p_{i} u_{j} d m$, we can write

$$
r_{i j}=\left(\lambda_{j}-\lambda_{i}\right) a_{i j} .
$$

It follows from (2.5), (2.6) and (2.7) that

$$
\begin{aligned}
\left(\lambda_{k+1}-\lambda_{i}\right)\left\|\phi_{i}\right\|^{2} & \leq \int_{\Omega} \phi_{i} \mathcal{L}^{2} \phi_{i} d m-\lambda_{i}\left\|\phi_{i}\right\|^{2} \\
& \leq \int_{\Omega} \phi_{i} p_{i} d m=w_{i}+\sum_{j=1}^{k}\left(\lambda_{i}-\lambda_{j}\right) a_{i j}^{2},
\end{aligned}
$$

where $w_{i}=\int_{\Omega} h u_{i} p_{i} d m$. We use that $\int_{\Omega} \phi_{i} u_{j} d m=0$ again to get

$$
\begin{aligned}
\left(\lambda_{k+1}-\lambda_{i}\right)\left(\int_{\Omega} \phi_{i} p_{i} d m\right)^{2} & \left.=\left(\lambda_{k+1}-\lambda_{i}\right)\right)\left(\int_{\Omega} \phi_{i}\left(p_{i}-\sum_{j=1}^{k} r_{i j} u_{j} d m\right)^{2}\right. \\
& \leq\left(\lambda_{k+1}-\lambda_{i}\right)\left\|\phi_{i}\right\|^{2}\left(\left\|p_{i}\right\|^{2}-\sum_{j=1}^{k} r_{i j}^{2}\right) \\
& \leq\left(\int_{\Omega} \phi_{i} p_{i} d m\right)\left(\left\|p_{i}\right\|^{2}-\sum_{j=1}^{k} r_{i j}^{2}\right)
\end{aligned}
$$

this implies that

$$
\left(\lambda_{k+1}-\lambda_{i}\right)\left(\int_{\Omega} \phi_{i} p_{i} d m\right) \leq\left\|p_{i}\right\|^{2}-\sum_{j=1}^{k} r_{i j}^{2}
$$

Multiplying (2.15) by $\left(\lambda_{k+1}-\lambda_{i}\right)$ and summing on $i$ from 1 to $k$, we obtain (2.16)

$$
\sum_{i=1}^{k}\left(\lambda_{k+1}-\lambda_{i}\right)^{2} \int_{\Omega} \phi_{i} p_{i} d m \leq \sum_{i=1}^{k}\left(\lambda_{k+1}-\lambda_{i}\right)\left\|p_{i}\right\|^{2}-\sum_{i, j=1}^{k}\left(\lambda_{k+1}-\lambda_{i}\right)\left(\lambda_{i}-\lambda_{j}\right)^{2} a_{i j}^{2} .
$$


Multiplying (2.13) by $\left(\lambda_{k+1}-\lambda_{i}\right)^{2}$, summing on $i$ from 1 to $k$ and $a_{i j}=a_{j i}$, we infer

$$
\begin{aligned}
\sum_{i=1}^{k}\left(\lambda_{k+1}-\lambda_{i}\right)^{2} \int_{\Omega} \phi_{i} p_{i} d m & =\sum_{i=1}^{k}\left(\lambda_{k+1}-\lambda_{i}\right)^{2} w_{i}+\sum_{i, j=1}^{k}\left(\lambda_{k+1}-\lambda_{i}\right)^{2}\left(\lambda_{i}-\lambda_{j}\right) a_{i j}^{2} \\
& =\sum_{i=1}^{k}\left(\lambda_{k+1}-\lambda_{i}\right)^{2} w_{i}-\sum_{i, j=1}^{k}\left(\lambda_{k+1}-\lambda_{i}\right)\left(\lambda_{i}-\lambda_{j}\right)^{2} a_{i j}^{2}
\end{aligned}
$$

then

$$
\sum_{i=1}^{k}\left(\lambda_{k+1}-\lambda_{i}\right)^{2} w_{i} \leq \sum_{i=1}^{k}\left(\lambda_{k+1}-\lambda_{i}\right)\left\|p_{i}\right\|^{2},
$$

which shows that (2.1) is true. In order to prove (2.2), we set

$$
b_{i j}=\int_{\Omega} u_{j}\left(T\left(\nabla h, \nabla u_{i}\right)+\frac{u_{i}}{2} \mathcal{L} h\right) d m .
$$

Observe that

$$
\begin{aligned}
b_{i j} & =-\int_{\Omega} u_{i} \operatorname{div}\left(u_{j} T \nabla h\right) d m+\frac{1}{2} \int_{\Omega} u_{i} u_{j} \mathcal{L} h d m \\
& =-\int_{\Omega} u_{i}\left(T\left(\nabla h, \nabla u_{j}\right)+\frac{u_{j}}{2} \mathcal{L} h\right) d m=-b_{j i},
\end{aligned}
$$

and

$$
-2 \int_{\Omega} \phi_{i}\left(T\left(\nabla h, \nabla u_{i}\right)+\frac{u_{i}}{2} \mathcal{L} h\right) d m=v_{i}+2 \sum_{j=1}^{k} a_{i j} b_{i j},
$$

where

$$
v_{i}=\int_{\Omega}\left(-h u_{i}^{2} \mathcal{L} h-2 h u_{i} T\left(\nabla h, \nabla u_{i}\right)\right) d m=\int_{\Omega} u_{i}^{2} T(\nabla h, \nabla h) d m .
$$

Since for any positive constant $\delta$ and for all $x, y \in \mathbb{R}$ we have $-2 x y \leq \delta x^{2}+\frac{y^{2}}{\delta}$, then multiplying (2.20) by $\left(\lambda_{k+1}-\lambda_{i}\right)^{2}$, for any positive constant $\delta$, we get

$$
\begin{aligned}
& \left(\lambda_{k+1}-\lambda_{i}\right)^{2}\left(v_{i}+2 \sum_{j=1}^{k} a_{i j} b_{i j}\right) \\
& =\left(\lambda_{k+1}-\lambda_{i}\right)^{2} \int_{\Omega}\left(-2 \phi_{i}\right)\left(T\left(\nabla h, \nabla u_{i}\right)+\frac{u_{i}}{2} \mathcal{L} h-\sum_{j=1}^{k} b_{i j} u_{j}\right) d m \\
& \leq \delta\left(\lambda_{k+1}-\lambda_{i}\right)^{3}\left\|\phi_{i}\right\|^{2}+\frac{1}{\delta}\left(\lambda_{k+1}-\lambda_{i}\right) \int_{\Omega}\left(T\left(\nabla h, \nabla u_{i}\right)+\frac{u_{i}}{2} \mathcal{L} h-\sum_{j=1}^{k} b_{i j} u_{j}\right) d m \\
& \leq \delta\left(\lambda_{k+1}-\lambda_{i}\right)^{3}\left\|\phi_{i}\right\|^{2}+\frac{1}{\delta}\left(\lambda_{k+1}-\lambda_{i}\right)\left(\left\|T\left(\nabla h, \nabla u_{i}\right)+\frac{u_{i}}{2} \mathcal{L} h\right\|^{2}-\sum_{j=1}^{k} b_{i j}^{2}\right),
\end{aligned}
$$


hence (2.13) implies that

$$
\begin{aligned}
\left(\lambda_{k+1}-\lambda_{i}\right)^{2}\left(v_{i}+2 \sum_{j=1}^{k} a_{i j} b_{i j}\right) \leq & \delta\left(\lambda_{k+1}-\lambda_{i}\right)^{2}\left(w_{i}+\sum_{j=1}^{k}\left(\lambda_{i}-\lambda_{j}\right) a_{i j}^{2}\right) \\
& +\frac{1}{\delta}\left(\lambda_{k+1}-\lambda_{i}\right)\left(\left\|T\left(\nabla h, \nabla u_{i}\right)+\frac{u_{i}}{2} \mathcal{L} h\right\|^{2}-\sum_{j=1}^{k} b_{i j}^{2}\right) .
\end{aligned}
$$

Now, summing over $i$ from 1 to $k, a_{i j}=a_{i j}$ and $b_{i j}=-b_{j i}$ we conclude that

$$
\begin{aligned}
& \sum_{i=1}^{k}\left(\lambda_{k+1}-\lambda_{i}\right)^{2} v_{i}-2 \sum_{i, j=1}^{k}\left(\lambda_{k+1}-\lambda_{i}\right)\left(\lambda_{i}-\lambda_{j}\right) a_{i j} b_{i j} \\
& \leq \sum_{i=1}^{k} \delta\left(\lambda_{k+1}-\lambda_{i}\right)^{2} w_{i}-\sum_{i, j=1}^{k} \delta\left(\lambda_{k+1}-\lambda_{i}\right)\left(\lambda_{i}-\lambda_{j}\right)^{2} a_{i j}^{2} \\
& +\frac{1}{\delta} \sum_{i=1}^{k}\left(\lambda_{k+1}-\lambda_{i}\right)\left\|T\left(\nabla h, \nabla u_{i}\right)+\frac{u_{i}}{2} \mathcal{L} h\right\|^{2}-\frac{1}{\delta} \sum_{i, j=1}^{k}\left(\lambda_{k+1}-\lambda_{i}\right) b_{i j}^{2},
\end{aligned}
$$

which gives

$$
\begin{aligned}
\sum_{i=1}^{k}\left(\lambda_{k+1}-\lambda_{i}\right)^{2} v_{i} \leq & \sum_{i=1}^{k} \delta\left(\lambda_{k+1}-\lambda_{i}\right)^{2} w_{i}+\frac{1}{\delta} \sum_{i=1}^{k}\left(\lambda_{k+1}-\lambda_{i}\right)\left\|T\left(\nabla h, \nabla u_{i}\right)+\frac{u_{i}}{2} \mathcal{L} h\right\|^{2} \\
(2.21) & -\sum_{i, j=1}^{k}\left(\lambda_{k+1}-\lambda_{i}\right)\left(\sqrt{\delta}\left(\lambda_{i}-\lambda_{j}\right) a_{i j}-\frac{1}{\delta} b_{i j}\right)^{2} \\
\leq & \sum_{i=1}^{k} \delta\left(\lambda_{k+1}-\lambda_{i}\right)^{2} w_{i}+\frac{1}{\delta} \sum_{i=1}^{k}\left(\lambda_{k+1}-\lambda_{i}\right)\left\|T\left(\nabla h, \nabla u_{i}\right)+\frac{u_{i}}{2} \mathcal{L} h\right\|^{2} .
\end{aligned}
$$

Thus (2.2) is true. Substituting (2.18) into (2.21) complete the proof of the proposition.

Proof of Theorem [1.1. Let $x_{1}, \ldots, x_{n}$ be the standard Euclidean coordinate of $\mathbb{R}^{m}$, $\bar{\nabla}$ be the Canonical connection of $\mathbb{R}^{m}$ and $\left\{e_{1}, \ldots, e_{m}\right\}$ be a local orthonormal geodesic frame in $p \in M$ adapted to $M$, then

$$
\bar{\nabla} x_{r}=\sum_{i=1}^{n} e_{i}\left(x_{r}\right) e_{i}+\sum_{i=n+1}^{m} e_{i}\left(x_{r}\right) e_{i}, \quad e_{r}=\nabla x_{r}+\left(\nabla x_{r}\right)^{\perp} .
$$

Therefore

$\sum_{r=1}^{m} T\left(\nabla x_{r}, \nabla u_{i}\right)=\sum_{r=1}^{m}\left\langle\nabla x_{r}, T\left(\nabla u_{i}\right)\right\rangle=\sum_{r=1}^{m}\left\langle e_{r}-\left(\nabla x_{r}\right)^{\perp}, T\left(\nabla u_{i}\right)\right\rangle=\sum_{r=1}^{m}\left\langle e_{r}, T\left(\nabla u_{i}\right)\right\rangle$,

and

$$
\sum_{r=1}^{m} T\left(\nabla x_{r}, \nabla u_{i}\right)^{2}=\sum_{r=1}^{m}\left\langle e_{r}, T\left(\nabla u_{i}\right)\right\rangle=\left|T\left(\nabla u_{i}\right)\right|^{2} .
$$


Also, we have

$(2.23)$

$$
\sum_{r=1}^{m} T\left(\nabla x_{r}, \nabla x_{r}\right)=\sum_{r=1}^{m}\left\langle e_{r}, T\left(\nabla x_{r}\right)\right\rangle=\sum_{r=1}^{m}\left\langle T\left(e_{r}\right), \nabla x_{r}\right\rangle=\sum_{r=1}^{m}\left\langle T\left(e_{r}\right), e_{r}\right\rangle=\operatorname{tr}(T) .
$$

For $x=\left(x_{1}, \ldots, x_{n}\right)$, we compute

$$
\begin{aligned}
\operatorname{div}(T(\nabla x)) & :=\left(\operatorname{div}\left(T\left(\nabla x_{1}\right)\right), \ldots, \operatorname{div}\left(T\left(\nabla x_{m}\right)\right)\right) \\
& =\left(\sum_{i=1}^{n} e_{i}\left\langle T\left(\nabla x_{1}\right), e_{i}\right\rangle, \ldots, \sum_{i=1}^{n} e_{i}\left\langle T\left(\nabla x_{m}\right), e_{i}\right\rangle\right) \\
& =\sum_{i, j=1}^{n}\left(e_{i}\left(e_{j}\left(x_{1}\right)\left\langle T\left(e_{j}\right), e_{i}\right\rangle\right), \ldots, e_{i}\left(e_{j}\left(x_{m}\right)\left\langle T\left(e_{j}\right), e_{i}\right\rangle\right)\right) \\
& =\sum_{i, j=1}^{n}\left(e_{i} e_{j}\left(x_{1}\right)\left\langle T\left(e_{j}\right), e_{i}\right\rangle, \ldots, e_{i} e_{j}\left(x_{m}\right)\left\langle T\left(e_{j}\right), e_{i}\right\rangle\right) \\
& +\sum_{i, j=1}^{n}\left(e_{j}\left(x_{1}\right)\left\langle\nabla_{e_{i}} T\left(e_{j}\right), e_{i}\right\rangle, \ldots, e_{j}\left(x_{m}\right)\left\langle\nabla_{e_{i}} T\left(e_{j}\right), e_{i}\right\rangle\right) \\
& =\sum_{i, j=1}^{n}\left\langle T\left(e_{j}\right), e_{i}\right\rangle \bar{\nabla}_{e_{i}} e_{j}(x)+\sum_{i, j=1}^{n}\left\langle\nabla_{e_{i}} T\left(e_{j}\right), e_{i}\right\rangle e_{j}(x) \\
& =\sum_{i, j=1}^{n}\left\langle T\left(e_{j}\right), e_{i}\right\rangle \alpha\left(e_{i}, e_{j}\right)(x)+\sum_{i, j=1}^{n}\left\langle\nabla_{e_{i}} T\left(e_{i}\right), e_{j}\right\rangle e_{j}(x) \\
& =\sum_{j=1}^{n} \alpha\left(T\left(e_{j}\right), e_{j}\right)(x)+\sum_{j=1}^{n} \nabla_{e_{i}} T\left(e_{i}\right)(x)=\operatorname{tr}(\alpha \circ T)(x)+\operatorname{tr}(\nabla T)(x),
\end{aligned}
$$

hence

$$
\sum_{r=1}^{m}\left(\operatorname{div}\left(T\left(\nabla x_{r}\right)\right)\right)^{2}=\|\operatorname{div} T(\nabla x)\|^{2}=\|\operatorname{tr}(\alpha \circ T)\|^{2}+|\operatorname{tr}(\nabla T)|^{2}
$$

and

$$
\begin{aligned}
\sum_{r=1}^{m} \operatorname{div}\left(T\left(\nabla x_{r}\right)\right) T\left(\nabla x_{r}, \nabla u_{i}\right) & =\sum_{r=1}^{m} \operatorname{div}\left(T\left(\nabla x_{r}\right)\right) T\left(\nabla u_{i}\right)\left(x_{r}\right) \\
& =\left\langle\operatorname{div}(T(\nabla x)), T\left(\nabla u_{i}\right)\right\rangle=\left\langle\operatorname{tr}(\nabla T), T\left(\nabla u_{i}\right)\right\rangle,
\end{aligned}
$$

where $\alpha \circ T=\alpha(T(),.) \in \mathcal{X}(M)^{\perp}$. By taking $h=x_{r}$ in (2.2) we can write

$$
\begin{aligned}
\sum_{i=1}^{k}\left(\lambda_{k+1}-\lambda_{i}\right)^{2} v_{i} \leq & \delta \sum_{i=1}^{k}\left(\lambda_{k+1}-\lambda_{i}\right)^{2} w_{i} \\
& +\frac{1}{\delta} \sum_{i=1}^{k}\left(\lambda_{k+1}-\lambda_{i}\right)\left\|T\left(\nabla x_{r}, \nabla u_{i}\right)+\frac{u_{i} \mathcal{L} x_{r}}{2}\right\|^{2} .
\end{aligned}
$$


where

$$
\begin{aligned}
w_{i} & =\int_{\Omega} x_{r} u_{i} p_{i} d m, \\
p_{i} & =\mathcal{L} x_{r} \mathcal{L} u_{i}+2 T\left(\nabla x_{r}, \nabla \mathcal{L} u_{i}\right)+\mathcal{L}\left(u_{i} \mathcal{L} h\right)+2 \mathcal{L} T\left(\nabla x_{r}, \nabla u_{i}\right), \\
v_{i} & =\int_{\Omega} u_{i}^{2} T\left(\nabla x_{r}, \nabla x_{r}\right) d m .
\end{aligned}
$$

Summing over $r$, we have

$$
\begin{aligned}
\sum_{r=1}^{m} \sum_{i=1}^{k}\left(\lambda_{k+1}-\lambda_{i}\right)^{2} v_{i} \leq & \delta \sum_{r=1}^{m} \sum_{i=1}^{k}\left(\lambda_{k+1}-\lambda_{i}\right)^{2} w_{i} \\
& +\frac{1}{\delta} \sum_{i=1}^{k}\left(\lambda_{k+1}-\lambda_{i}\right) \sum_{r=1}^{m}\left\|T\left(\nabla x_{r}, \nabla u_{i}\right)+\frac{u_{i} \mathcal{L} x_{r}}{2}\right\|^{2},
\end{aligned}
$$

and from (2.23) we get

$$
\sum_{r=1}^{m} v_{i}=\sum_{r=1}^{m} \int_{\Omega} u_{i}^{2} T\left(\nabla x_{r}, \nabla x_{r}\right) d m=\int_{\Omega} u_{i}^{2} \operatorname{tr}(T) d m .
$$

Also (2.25) and (2.24) imply that

$$
\begin{aligned}
\sum_{r=1}^{m} \int_{\Omega} x_{r} u_{i} \mathcal{L} x_{r} \mathcal{L} u_{i} d m & =\int_{\Omega} u_{i} \mathcal{L} u_{i}\langle\operatorname{div} T(\nabla x), x\rangle d m \\
& =\int_{\Omega} u_{i} \mathcal{L} u_{i}\langle\operatorname{tr}(\alpha \circ T)+\operatorname{tr}(\nabla T), I\rangle d m
\end{aligned}
$$

$$
\begin{aligned}
& \text { 31) } \\
& \sum_{r=1}^{m} \int_{\Omega} x_{r} u_{i} \mathcal{L}\left(u_{i} \mathcal{L} x_{r}\right) d m \\
= & \sum_{r=1}^{m} \int_{\Omega} \mathcal{L}\left(x_{r} u_{i}\right) u_{i} \mathcal{L} x_{r} d m \\
= & \sum_{r=1}^{m} \int_{\Omega}\left(x_{r} u_{i} \mathcal{L} u_{i} \mathcal{L} x_{r}+u_{i}^{2}\left(\mathcal{L} x_{r}\right)^{2}+2 T\left(\nabla u_{i}, \nabla x_{r}\right) u_{i} \mathcal{L} x_{r}\right) d m \\
= & \int_{\Omega} u_{i} \mathcal{L} u_{i}\langle\operatorname{tr}(\alpha \circ T)+\operatorname{tr}(\nabla T), I\rangle d m+\int_{\Omega} u_{i}^{2}\left(\|\operatorname{tr}(\alpha \circ T)\|^{2}+|\operatorname{tr}(\nabla T)|^{2}\right) d m \\
& +2 \int_{\Omega} u_{i}\left\langle T\left(\nabla u_{i}\right), \operatorname{tr}(\nabla T)\right\rangle d m,
\end{aligned}
$$


and

$$
\begin{aligned}
& \sum_{r=1}^{m} \int_{\Omega} 2 x_{r} u_{i} \mathcal{L} T\left(\nabla x_{r}, \nabla u_{i}\right) d m \\
= & 2 \sum_{r=1}^{m} \int_{\Omega} \mathcal{L}\left(x_{r} u_{i}\right) T\left(\nabla x_{r}, \nabla u_{i}\right) d m \\
= & 2 \sum_{r=1}^{m} \int_{\Omega} u_{i} \mathcal{L} x_{r} T\left(\nabla x_{r}, \nabla u_{i}\right) d m+2 \sum_{r=1}^{m} \int_{\Omega} x_{r} \mathcal{L} u_{i} T\left(\nabla x_{r}, \nabla u_{i}\right) d m \\
& +2 \sum_{r=1}^{m} \int_{\Omega} T\left(\nabla x_{r}, \nabla u_{i}\right)^{2} d m \\
= & 2 \int_{\Omega} u_{i}\left\langle T\left(\nabla u_{i}\right), \operatorname{tr}(\nabla T)\right\rangle d m+4 \int_{\Omega}\left|T\left(\nabla u_{i}\right)\right|^{2} d m+2 \int_{\Omega} \mathcal{L} u_{i}\left\langle T\left(\nabla u_{i}\right), I\right\rangle d m
\end{aligned}
$$

$$
\sum_{r=1}^{m} \int_{\Omega} 2 x_{r} u_{i} T\left(\nabla x_{r}, \nabla \mathcal{L} u_{i}\right) d m=2 \int_{\Omega} u_{i}\left\langle T\left(\nabla \mathcal{L} u_{i}\right), I\right\rangle d m
$$

Thus

$$
\begin{aligned}
\sum_{r=1}^{m} w_{i}= & 2 \int_{\Omega} u_{i} \mathcal{L} u_{i}\langle\operatorname{tr}(\alpha \circ T)+\operatorname{tr}(\nabla T), I\rangle d m+2 \int_{\Omega} u_{i}\left\langle T\left(\nabla \mathcal{L} u_{i}\right), I\right\rangle d m \\
(2.34) \quad & +\int_{\Omega} u_{i}^{2}\left(\|\left.\operatorname{tr}(\alpha \circ T)\right|^{2}+|\operatorname{tr}(\nabla T)|^{2}\right) d m+4 \int_{\Omega} u_{i}\left\langle T\left(\nabla u_{i}\right), \operatorname{tr}(\nabla T)\right\rangle d m \\
& +4 \int_{\Omega}\left|T\left(\nabla u_{i}\right)\right|^{2} d m+2 \int_{\Omega} \mathcal{L} u_{i}\left\langle T\left(\nabla u_{i}\right), I\right\rangle d m
\end{aligned}
$$

and

$$
\begin{aligned}
& (2.35) \sum_{r=1}^{m}\left\|T\left(\nabla x_{r}, \nabla u_{i}\right)+\frac{u_{i} \mathcal{L} x_{r}}{2}\right\|^{2} \\
& =\sum_{r=1}^{m} \int_{\Omega}\left(T\left(\nabla x_{r}, \nabla u_{i}\right)^{2}+u_{i} \mathcal{L} x_{r} T\left(\nabla x_{r}, \nabla u_{i}\right)+\frac{1}{4} u_{i}^{2}\left(\mathcal{L} x_{r}\right)^{2}\right) d m \\
& =\int_{\Omega}\left(\left|T\left(\nabla u_{i}\right)\right|^{2}+u_{i}\left\langle T\left(\nabla u_{i}\right), \operatorname{tr}(\nabla T)\right\rangle+\frac{1}{4} u_{i}^{2}\left(\|\operatorname{tr}(\alpha \circ T)\|^{2}+|\operatorname{tr}(\nabla T)|^{2}\right)\right) d m .
\end{aligned}
$$

Substituting (2.29), (2.34) and (2.35) into (2.27) we complete the proof of the theorem. 
Proof of Theorem 1.2. Let $S_{e_{i}}$ be the Weingarten operator of the immersion with respect to $e_{i}$. Then

$$
\begin{aligned}
\|t r(\alpha \circ T)\|^{2} & =\left\|\sum_{i=1}^{n} \alpha\left(T e_{i}, e_{i}\right)\right\|^{2}=\left\|\sum_{i=1}^{n} \sum_{k=n+1}^{m}\left\langle\alpha\left(T e_{i}, e_{i}\right), e_{k}\right\rangle e_{k}\right\|^{2} \\
& =\left\|\sum_{k=n+1}^{m}\left(\sum_{i=1}^{n}\left\langle S_{e_{k}} e_{i}, T e_{i}\right\rangle\right) e_{k}\right\|^{2}=\left\|\sum_{k=n+1}^{m}\left\langle S_{e_{k}}, T\right\rangle e_{k}\right\|^{2} \\
& \leq \sum_{k=n+1}^{m}\left|\left\langle S_{e_{k}}, T\right\rangle\right|^{2} \sum_{k=n+1}^{m}\left|e_{k}\right|^{2} \leq \sum_{k=n+1}^{m}\left|S_{e_{k}}\right|^{2}|T|^{2} \sum_{k=n+1}^{m}\left|e_{k}\right|^{2} \\
& \leq(m-n) S_{0}^{2} T_{*}^{2},
\end{aligned}
$$

where $S_{0}=\max \left\{\sup _{\bar{\Omega}}\left|S_{e_{k}}\right|: k=n+1, \ldots, m\right\}$ and $T_{*}=\sup _{\bar{\Omega}}|T| . \quad$ If $T_{0}=$ $\sup _{\bar{\Omega}}|\operatorname{tr}(\nabla T)|$ and $I_{0}=\sup _{\bar{\Omega}}|I|$ then

$$
\begin{aligned}
\int_{\Omega} u_{i}\left\langle T\left(\nabla u_{i}\right), \operatorname{tr}(\nabla T)\right\rangle d m & \leq\left(\int_{\Omega} u_{i}^{2} d m\right)^{\frac{1}{2}}\left(\int_{\Omega}\left|T\left(\nabla u_{i}\right)\right|^{2}|\operatorname{tr}(\nabla T)|^{2} d m\right)^{\frac{1}{2}} \\
& \leq T_{0}\left\|T\left(\nabla u_{i}\right)\right\|_{L^{2}(\Omega)},
\end{aligned}
$$

$$
\begin{aligned}
& \int_{\Omega} u_{i} \mathcal{L} u_{i}\langle\operatorname{tr}(\alpha \circ T)+\operatorname{tr}(\nabla T), I\rangle d m \\
& =\int_{\Omega} u_{i} \mathcal{L} u_{i}\langle\operatorname{tr}(\alpha \circ T), I\rangle d m+\int_{\Omega} u_{i} \mathcal{L} u_{i}\langle\operatorname{tr}(\nabla T), I\rangle d m \\
& \leq\left(\int_{\Omega} u_{i}^{2} d m\right)^{\frac{1}{2}}\left(\left.\int_{\Omega}\left(\mathcal{L} u_{i}\right)^{2}|| \operatorname{tr}(\alpha \circ T)\right|^{2}|I|^{2} d m\right)^{\frac{1}{2}} \\
& +\left(\int_{\Omega} u_{i}^{2} d m\right)^{\frac{1}{2}}\left(\int_{\Omega}\left(\mathcal{L} u_{i}\right)^{2}|\operatorname{tr}(\nabla T)|^{2}|I|^{2} d m\right)^{\frac{1}{2}} \\
& \leq\left(\sqrt{m-n} S_{0} T_{*}+T_{0}\right) I_{0} \lambda_{i}^{\frac{1}{2}}
\end{aligned}
$$

and

$$
\begin{aligned}
\int_{\Omega} u_{i}\left\langle T\left(\nabla \mathcal{L} u_{i}\right), I\right\rangle d m & \leq\left(\int_{\Omega} u_{i}^{2} d m\right)^{\frac{1}{2}}\left(\int_{\Omega}\left|T\left(\nabla \mathcal{L} u_{i}\right)\right|^{2}|I|^{2} d m\right)^{\frac{1}{2}} \\
& \leq I_{0}\left\|T\left(\nabla \mathcal{L} u_{i}\right)\right\|_{L^{2}(\Omega)} .
\end{aligned}
$$

Also, we have

$$
\int_{\Omega} u_{i}^{2}\left(\|t r(\alpha \circ T)\|^{2}+|\operatorname{tr}(\nabla T)|^{2}\right) d m \leq(m-n) S_{0}^{2} T_{*}^{2}+T_{0}^{2}
$$

and

$$
\begin{aligned}
\int_{\Omega} \mathcal{L} u_{i}\left\langle T\left(\nabla u_{i}\right), I\right\rangle d m & \leq\left(\int_{\Omega}\left(\mathcal{L} u_{i}\right)^{2} d m\right)^{\frac{1}{2}}\left(\int_{\Omega}\left|T\left(\nabla u_{i}\right)\right|^{2}|I|^{2} d m\right)^{\frac{1}{2}} \\
& \leq \lambda_{i} I_{0}\left\|T\left(\nabla u_{i}\right)\right\|_{L^{2}(\Omega)} .
\end{aligned}
$$

By setting

$$
\begin{aligned}
C_{i}= & 2\left(\sqrt{m-n} S_{0} T_{*}+T_{0}\right) I_{0} \lambda_{i}^{\frac{1}{2}}+I_{0}\left\|T\left(\nabla \mathcal{L} u_{i}\right)\right\|_{L^{2}(\Omega)}+(m-n) S_{0}^{2} T_{*}^{2}+T_{0}^{2} \\
& +4 T_{0}\left\|T\left(\nabla u_{i}\right)\right\|_{L^{2}(\Omega)}+4\left\|T\left(\nabla u_{i}\right)\right\|_{L^{2}(\Omega)}+2 \lambda_{i} I_{0}\left\|T\left(\nabla u_{i}\right)\right\|_{L^{2}(\Omega)},
\end{aligned}
$$


and

$$
D_{i}=\left\|T\left(\nabla u_{i}\right)\right\|_{L^{2}(\Omega)}+T_{0}\left\|T\left(\nabla u_{i}\right)\right\|_{L^{2}(\Omega)}+\frac{1}{4}\left((m-n) S_{0}^{2} T_{*}^{2}+T_{0}^{2}\right)
$$

we get $A_{i} \leq C_{i}$ and $B_{i} \leq D_{i}$. Substituting these inequalities into Theorem 1.1 we complete the proof of the Theorem.

Proof of Theorem 1.3. Taking $T$ equal to identity in Theorem 1, we obtain

$$
n \sum_{i=1}^{k}\left(\lambda_{k+1}-\lambda_{i}\right)^{2} \leq \delta \sum_{i=1}^{k}\left(\lambda_{k+1}-\lambda_{i}\right)^{2} E_{i}+\frac{1}{\delta} \sum_{i=1}^{k}\left(\lambda_{k+1}-\lambda_{i}\right) F_{i},
$$

where

$$
\begin{aligned}
E_{i}= & 2 \int_{\Omega} u_{i} \Delta u_{i}\langle n H, I\rangle d m+2 \int_{\Omega} u_{i}\left\langle\nabla \Delta u_{i}, I\right\rangle d m \\
& +\int_{\Omega} u_{i}^{2} n^{2}\|H\|^{2} d m+4 \int_{\Omega}\left|\nabla u_{i}\right|^{2} d m+2 \int_{\Omega} \Delta u_{i}\left\langle\nabla u_{i}, I\right\rangle d m
\end{aligned}
$$

and

$$
F_{i}=\int_{\Omega}\left\{\left|\nabla u_{i}\right|^{2}+\frac{u_{i}^{2}}{4} n^{2}\|H\|^{2}\right\} d m .
$$

On the other hand, we have

$$
\int_{\Omega} u_{i}\left\langle\nabla \Delta u_{i}, I\right\rangle d m=-\int_{\Omega} \Delta u_{i}\left\langle\nabla u_{i}, I\right\rangle d m
$$

and

$$
\begin{aligned}
\int_{\Omega}\left|\nabla u_{i}\right|^{2} d m & =\int_{\Omega}\left\langle\nabla u_{i}, \nabla u_{i}\right\rangle d m=-\int_{\Omega} u_{i} \Delta u_{i} d m \\
& \leq \int_{\Omega}\left|u_{i}\right|\left|\Delta u_{i}\right| d m \leq\left(\int_{\Omega} u_{i}^{2} d m\right)^{\frac{1}{2}}\left(\int_{\Omega}\left(\Delta u_{i}\right)^{2} d m\right)^{\frac{1}{2}} \leq \lambda_{i}^{\frac{1}{2}} .
\end{aligned}
$$

Hence

and

$$
E_{i} \leq 2 n H_{0} I_{0} \lambda_{i}^{\frac{1}{2}}+n^{2} H_{0}^{2}+4 \lambda_{i}^{\frac{1}{2}}
$$

$$
F_{i} \leq \lambda_{i}^{\frac{1}{2}}+\frac{1}{4} n^{2} H_{0}^{2}
$$

where $H_{0}=\sup _{\bar{\Omega}}|H|$ and $I_{0}=\sup _{\bar{\Omega}}|I|$. Substituting these inequality into (2.40) we complete the proof of the theorem.

Proof of Corollary 1.4. For a minimal hypersurface we have $H=0$, therefore Theorem 3 results that

$$
n \sum_{i=1}^{k}\left(\lambda_{k+1}-\lambda_{i}\right)^{2} \leq 4 \delta \sum_{i=1}^{k}\left(\lambda_{k+1}-\lambda_{i}\right)^{2} \lambda_{i}^{\frac{1}{2}}+\frac{1}{\delta} \sum_{i=1}^{k}\left(\lambda_{k+1}-\lambda_{i}\right) \lambda_{i}^{\frac{1}{2}}
$$

Taking

we get

$$
\delta=\left\{\frac{\sum_{i=1}^{k}\left(\lambda_{k+1}-\lambda_{i}\right) \lambda_{i}^{\frac{1}{2}}}{4 \sum_{i=1}^{k}\left(\lambda_{k+1}-\lambda_{i}\right)^{2} \lambda_{i}^{\frac{1}{2}}}\right\}^{\frac{1}{2}}
$$

$$
\frac{n}{4} \sum_{i=1}^{k}\left(\lambda_{k+1}-\lambda_{i}\right)^{2} \leq\left\{\sum_{i=1}^{k}\left(\lambda_{k+1}-\lambda_{i}\right)^{2} \lambda_{i}^{\frac{1}{2}} \sum_{i=1}^{k}\left(\lambda_{k+1}-\lambda_{i}\right) \lambda_{i}^{\frac{1}{2}}\right\}^{\frac{1}{2}} .
$$


On the other hand

$\left(\sum_{i=1}^{k}\left(\lambda_{k+1}-\lambda_{i}\right)^{2} \lambda_{i}^{\frac{1}{2}}\right)\left(\sum_{i=1}^{k}\left(\lambda_{k+1}-\lambda_{i}\right) \lambda_{i}^{\frac{1}{2}}\right) \leq\left(\sum_{i=1}^{k}\left(\lambda_{k+1}-\lambda_{i}\right)^{2}\right)\left(\sum_{i=1}^{k}\left(\lambda_{k+1}-\lambda_{i}\right) \lambda_{i}\right)$.

It and (2.42) imply that

$$
\sum_{i=1}^{k}\left(\lambda_{k+1}-\lambda_{i}\right)^{2} \leq \frac{16}{n^{2}} \sum_{i=1}^{k}\left(\lambda_{k+1}-\lambda_{i}\right) \lambda_{i}
$$

solving this quadratic polynomial of $\lambda_{k+1}$, we obtain (1.7) and (1.8).

\section{REFERENCES}

[1] Q. M. Cheng, H. C. Yang, Inequalities for eigenvalues of a clamped plate problem, Trans. Amer. Soc. 358(2006) 2625-2635.

[2] G. N. Hile, R. Z. Yeh, Inequality for eigenvalues of the biharmonic operator, Pacific J. Math. 112(1984)115-133.

[3] S. M. Hook, Domain independent upper bounds for eigenvalues of elliptic operator, Trans. Amer. Math. Soc. 318(1990) 615-642.

[4] L. E. Payne, G. Pólya, H. F. Weinberger, On the ratio of consecutive eigenvalues, J. Math. Phys. 35( 1956) 289-298.

[5] Q. Wang, C. Xia, Universal bounds for eigenvalues of the biharmonic operator on Riemannian manifolds, Journal of functional analysis, 245 (2007) 334-352.

Department of Mathematics, Faculty of Sciences, Imam Khomeini International UniVERSiTy, QAZVIN, Iran.

E-mail address: azami@sci.ikiu.ac.ir 